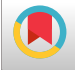

\title{
Faster Evaluation of ABCD Rule and Total Dermoscopic Score for Nevomelanocytic Lesions: Dermoscopic Score Scale
}

\author{
Betul Tas (iD) ${ }^{1, *}$ \\ ${ }^{1}$ Department of Dermatology and Venereology, Istanbul Bagcilar Research and Training Hospital, University of Health Sciences, Istanbul, Turkey \\ "Corresponding author: Department of Dermatology and Venereology, Istanbul Bagcilar Research and Training Hospital, University of Health Sciences, Istanbul, Turkey. Tel: \\ +90-2124404040, Email: betulavc@yahoo.com
}

Received 2020 February 23; Accepted 2020 March 09.

\begin{abstract}
Background: Dermoscopy is a dermatological examination method that is made with a special magnifying device called dermoscope (or dermatoscope). The device is usually used in the evaluation of nevomelanocytic lesions, and it is especially important in determining A, B, C, and D scores used in the calculation of "total dermoscopic score (TDS)". This score facilitates the identification of malignancy risk of lesions. For this evaluation, there is a need for several mathematical calculations, which are quite time-consuming.

Objectives: This study aimed to develop a new scale to calculate the score quicker to accelerate the determination of malignancy risk of nevomelanocytic lesions.

Methods: All possible scores, which were obtained by the addition of individually multiplying all possible values of A, B, C, and D letters by their own coefficients, were mathematically calculated with a simple calculator. The quartets of $A B C D$ giving the same score were reduced to their lowest numbers. All possible $A B$ and $C D$ pairs were systematically arranged on a scale that had a column of $\mathrm{AB}$ and a row of $\mathrm{CD}$ values. All results of the quartets were gradually placed on a scale from minimum to maximum.

Results: A total of 810 quartets of ABCD were determined. By the reduction of ones giving the same scores, a total of 207 values were obtained. All quartets and their total scores were placed on a scale called "dermoscopic score scale". On the scale, white, yellow, and red score areas indicated benign, suspicious, and high-risk nevomelanocytic lesions for melanoma.

Conclusions: This scale helps faster obtain TDS of nevomelanocytic lesions by decreasing the calculation period, especially when an analog handheld dermoscope is used.
\end{abstract}

Keywords: Dermoscopy, Diagnostic Techniques, Mathematical Concepts, Melanocytic Lesions, Risk Assessment

\section{Background}

Dermoscopy (DS) is an intravital and noninvasive examination method that analyses epidermis, dermoepidermal junction, and upper dermis of the alive skin using a glass plate, an immersion oil, and an epi-illumination by a tool called "dermoscope". Through this method, skin lesions can be examined at a 10 - 40-fold magnification (1, 2 ). Unna first described the use of a dermoscope with immersion oil in 1863, and called it "diascopy". Pehamberger et al. introduced the term "epiluminescent microscopy" into the literature in 1987, and developed the method "pattern analysis". The term "dermoscopy" was first introduced into the literature by Friedman et al. in 1991, and is still the most widely used method (3). Because dermoscopy requires training and experience, a few simplified algorithms have been developed until now for the dermoscopic diagnosis of pigmented skin lesions. They include ABCD rule, Menzies method, seven-point checklist (Argenziano method), and three-point checklist algorithm.

The ABCD rule was described by Stolz and Nachbar in 1994 , and is still one of the most used methods $(2,4)$. In this method, after a melanocytic lesion is examined clinically with the naked eye, the A, B, C, and D values are determined in the dermoscopic examination. By summing up the obtained values from the ABCD algorithm, a total score is calculated that is called total dermoscopic score(TDS). The calculation of TDS needs a few mathematical operations such as four multiplications and one addition (5). This evaluation usually takes a lot of time, especially in the case of the presence of multiple lesions. Although there are more complex dermoscopy devices that provide a shorter examination time and have digital imaging capabilities, they can cost several thousand dollars $(4,5)$. 


\section{Objectives}

This study aimed to develop a new and practical scale to calculate TDS quicker and practically to accelerate the determination of malignancy risk of nevomelanocytic lesions.

\section{Methods}

\subsection{Description of ABCD Algorithm}

To calculate TDS, one of the most used methods is the $\mathrm{ABCD}$ algorithm, in which each letter indicates a different feature of the lesion: "A" refers to asymmetry (regarding shape, color, and dermoscopic features), "B" to border irregularity (assuming the lesion is an eight-slice circle), " $\mathrm{C}$ " to color (one point for each different color), and " $\mathrm{D}$ " to differentiated structures (one point for each different structure such as pigment network, structureless area(s), dot(s), globule(s), and branched streak(s) $(2,4)$.

\subsection{Determination of TDS}

In this calculation, each letter can bring a different number of values: three for A, eight for B, six for C, and five for D. Each parameter has different coefficients: 1.3 for A, 0.1 for $\mathrm{B}$, and 0.5 for $\mathrm{C}$ and $\mathrm{D}$. We obtain TDS by the addition of individually multiplying all possible values of each letter by their own coefficients. The minimum score obtained is 1 whereas the maximum score is 8.9. ATDS $\leq 4.75$ indicates benign melanocytic lesion, a TDS of $4.8-5.45$ indicates a suspicious lesion, and a TDS $>5.45$ suggests a high likelihood of melanoma $(2,4,5)$ (Table 1$)$.

Patients who have TDS between 4.8 and 5.45 are recommended that they should be re-examined at regular intervals against the possibility of developing melanoma, and ones having TDS of higher than 5.45 are recommended that they should be operated for examining of their lesions histopathologically (5). A suspicious melanocytic lesion without criteria suggestive of melanoma should be monitored in short-term follow-ups within three to four months (6). On the other hand, patients having known risk factors for melanoma but not having any suspicion in their lesions can be monitored using long-term follow-ups within six to 12 months (7).

3.3. Mathematical Analysis to Create the Dermoscopic Score Scale

First, all possible TDSs that were obtained by the addition of individually multiplying all possible values of each letter by their own coefficients were calculated with a simple calculator (Casio ${ }^{\circledR}$ DJ-240D PLUS, 300 steps check, 14 digits, Casio Computer Co., Ltd., Shibuya, Tokyo, Japan), using a classical scoring formula as shown below (2-5).
$T D S=(A \times 1.3)+(B \times 0.1)+(C \times 0.5)+(D \times 0.5)$

Then, all possible quad combinations of ABCD giving these results were determined. The counts of quartets giving the same TDS were reduced to their lowest numbers. All AB (e.g., 00, 01, 02, 03, etc.) and CD (e.g., 11, 12, 13, 14, etc.) pairs were systematically arranged on a graduated scale consisting of a column of $\mathrm{AB}$ values and a row of $\mathrm{CD}$ values. Finally, all possible TDS results were placed on this scale from smallest to biggest, which were ordered left to right and top to bottom, respectively. In this scale, TDSs $\leq 4.75$ (benign) were placed in the white area, TDSs between 4.8 - 5.45 (suspicious for malignancy) in the yellow area, and TDSs $>5.45$ (favoring melanoma) in the red area.

\subsection{Ethical Issues}

The present study was conducted after the endorsement of the local Ethics Committee of the Bagcilar Research and Training Hospital of the University of Health Sciences (approval code: 10600/11/19). As the study was mathematical research that used no live subjects, no consent was required.

\section{Results}

The total number of possible $\mathrm{AB}$ pairs was 27 , whereas the total number of $C D$ pairs was 30 . With computer calculations, the total number of all possible quad combinations of values of $\mathrm{A}, \mathrm{B}, \mathrm{C}$, and D letters, as well as the total number of TDS, was $810(30 \times 27=810)$. Some combinations of $A B C D$ quartets gave the same TDS. For example, the total score of A1B1C1D5, A1B1C2D4, and A1B1C3D3 combinations was 4.4, while the total score of A2B4C4D5, A2B4C5D4, and $\mathrm{A} 2 \mathrm{~B} 4 \mathrm{C} 6 \mathrm{D} 3 \mathrm{combinations}$ was 7.5. In other words, some of the total $30 \mathrm{CD}$ pairs gave the same TDS results $(n=28)$ when treated with the same $A B$ pairs, except for the pairs of C1D1 and C6D5. To create only one CD row (surrounded by black), a simplification was performed by subtracting 20 pairs that gave the same TDS (surrounded by red) from the 28 ones, and 10 CD pairs [C1D1, C6D5, and $28-20=8$ pairs] left behind (top CD row). Finally, the $C D$ pairs giving the same results were placed on the top of each other (shown as eight columns marked in different colors) (Figure 1).

With this simplification, a total of $27 \mathrm{AB}$ and $10 \mathrm{CD}$ pairs and a total of 270 TDSs were obtained. Later, a scale with 27 $\mathrm{AB}$ columns and $10 \mathrm{CD}$ rows was created and the obtained TDSs were arranged on this scale systematically. It was called the Dermoscopic Score Scale (DSS). All possible values of both $\mathrm{AB}$ and $\mathrm{CD}$ pairs, as well as all possible TDS values, were shown in DSS from the smallest (1) to biggest (8.9) (Figure 2). On this colored scale, scores in white areas indicated benign nevomelanocytic lesions, yellow ones indicated suspicious nevomelanocytic lesions for malignancy 


\begin{tabular}{|c|c|c|c|}
\hline ABCD, Features & Value & Weight Factor & Subscores \\
\hline Asymmetry (regarding shape, color, and dermoscopic features) & & 1.3 & $0-2.6$ \\
\hline Symmetric & 0 & & \\
\hline One-axis asymmetry & 1 & & \\
\hline Two-axis asymmetry & 2 & & \\
\hline Border irregularity & & 0.1 & $0.5-3$ \\
\hline 0 to 8 -border & $0-8$ & & \\
\hline Color (one point for each color) & & 0.5 & $0.5-3$ \\
\hline White, light brown, dark brown, blue-grey, red, black & $1-6$ & & \\
\hline Differentiated structures (one point for each different structure) & & 0.5 & $0.5-2.5$ \\
\hline Pigment network, structureless area(s), dot(s), globule(s), branched streak(s) & $1-5$ & & \\
\hline TDS & & & $1.0-8.9$ \\
\hline
\end{tabular}

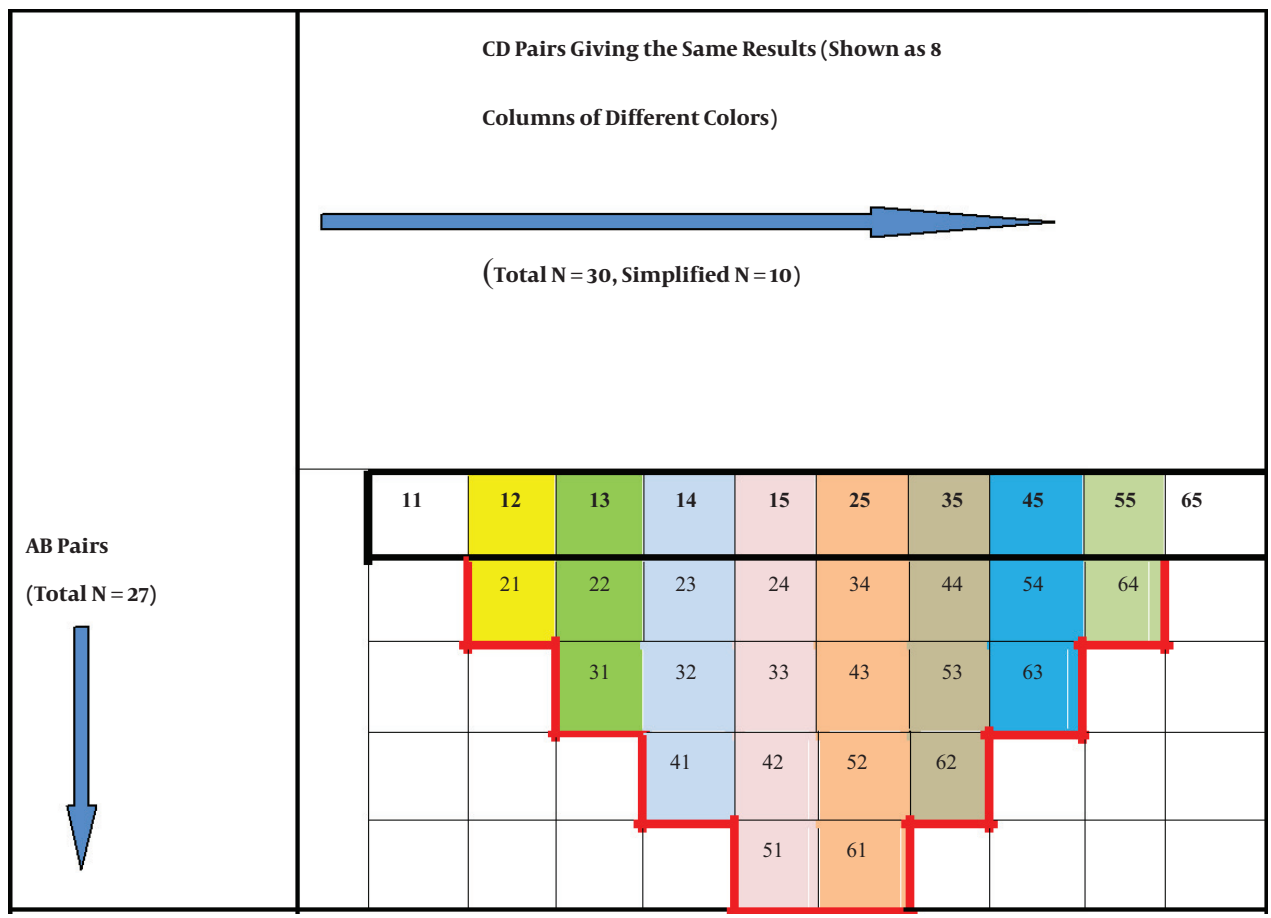

Figure 1. Columns of $\mathrm{CD}$ pairs giving the same results

requiring strict follow-up, and red ones indicated a high likelihood of melanoma.

\section{Discussion}

The time for a whole skin examination (WSE) includes the time necessary for both general visual examination and any required special examinations. The length of this time spent with patients is important in several aspects. The patients are more satisfied when their physicians spend more time on their exams and they feel that it was worth the time that they spend on telling their concerns. On the other hand, the reimbursement of the physician is indirectly related to the time required for a physician-patient interview. Gross et al. stated that a very short (one minute) estimate of the time was required for 


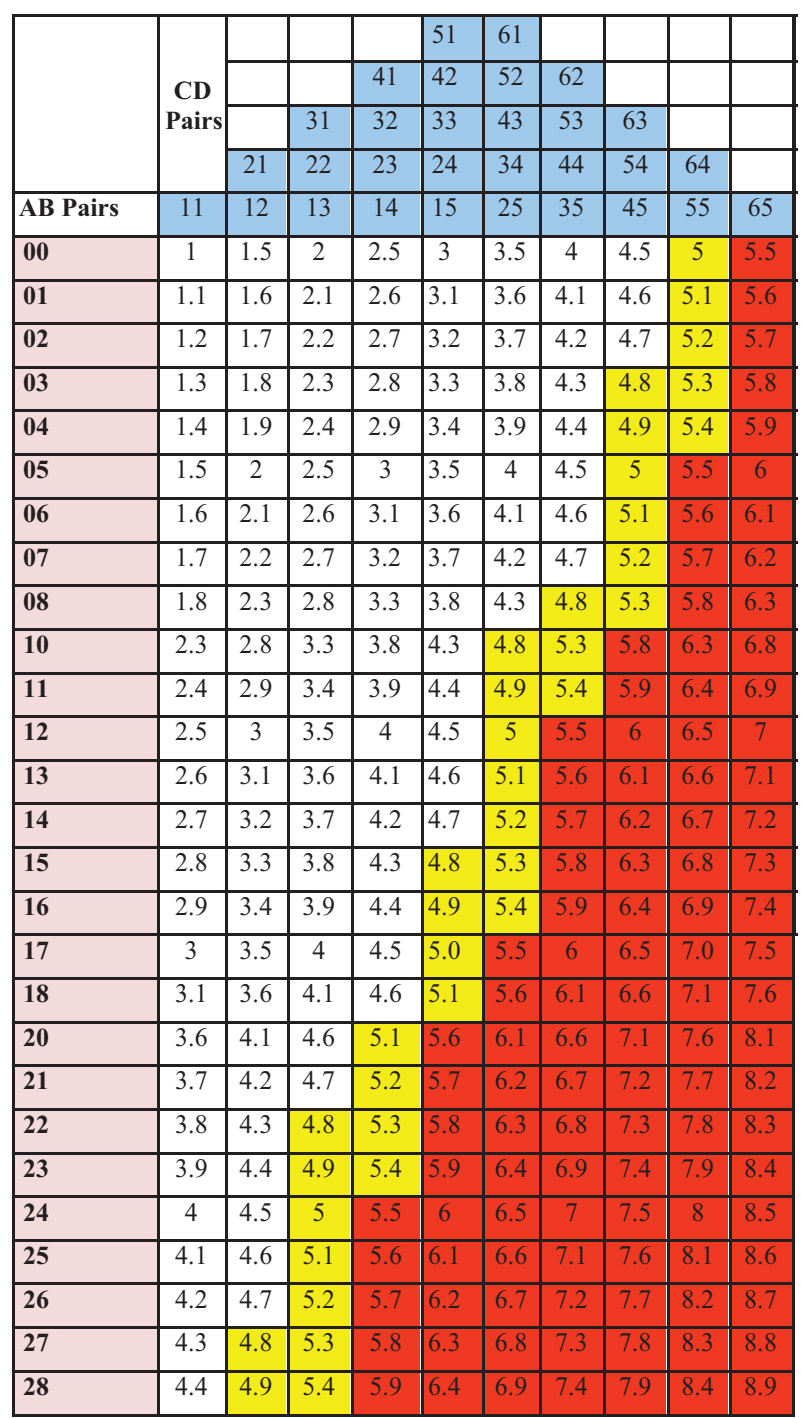

Figure 2. Dermoscopic score scale

a WSE (8). Also, Zalaudek et al. indicated in their multicenter study that the median time needed to complete a WSE without dermoscopy was 70 seconds. However, they added that a WSE aided by dermoscopy was significantly longer (142 seconds) than a WSE without dermoscopy, and a thorough WSE, with or without dermoscopy, required less than three minutes, which is a reasonable amount of added time to potentially prevent morbidity and mortality associated with skin cancer (9). Jaimes et al. suggested that the common reason for the precluding use of a dermoscope was time pressure. They also stated that the time for dermoscopy was a process depending on the performing operator (10). The time was reported as approximately seven minutes per patient and about 15 seconds per lesion by Kofler et al. (11). Additionally, a complete dermatological examination period includes additional procedures, such as Wood's lamp examination, potassium hydroxide preparation, swabs for bacterial/viral cultures, etc. $(8,9)$. It has been stated that dermoscopy will increase this time even more as part of the whole skin examination $(9,11,12)$, besides taking anamnesis, patient's undressing, or counseling $(8,12)$. Considering some factors such as the patient's age, tool use, scalp, genital, and mucous membrane examinations, the examination time will be more longer (12). In another study, it was stated that when making the whole dermatological assessment to reach a preliminary diagnosis, the physician should consider both clinical information such as the age of the patient, skin type, nevi counts, damage of ultraviolet radiation to the skin, famil$\mathrm{ial} /$ personal history of melanoma, and dermoscopic examination, besides the detection of special signs in the lesion, such as ugly duckling sign (13) and changes over time in repeated examinations (14). Another factor determining the dermoscopic examination time is differences in the experience of physicians. When it is performed by a more experienced expert, dermoscopic patterns are recognized more easily and the lesion is diagnosed faster than when it is done by less experienced physicians (15). Jaimes et al. pointed out another important factor for dermoscopic examination time, which is the bedside-examination of the patient that is a more uncomfortable and anxious situation than the ones made based on photographs. They stated that this condition may affect the decision-making, based on the fact that the assessment is made under the pressure of time (10). Digital dermoscopy is the gold standard for dermoscopic examination and its use for monitoring has proven to be the most effective method in the following of nevomelanocytic lesions and the early detection of melanomas (16). The reason is that this method makes it possible to conduct the photo documentation of images to use for comparison with other images taken in the follow-up periods. The most subtle changes in a lesion can be detected and evaluated with larger magnifications (17). When a digital one is used, the dermoscopic examination, which is performed at a single moment during a medical examination, takes a rather short time than examinations made by a handheld (or analog) device. However, although a computerized dermoscope provides a shorter examination time and has digital imaging capabilities, it can cost several thousand dollars $(5,18)$. Its other disadvantage is the lack of compliance. Many patients return for the next examination after much longer periods than the recommended intervals, and this cannot be underestimated. For the reason of the uncertainty of follow-ups, clinicians often feel the pressure to use more aggressive management such as redundant excisions. It has been stated that one of the most probable reasons for the lack of compliance is the 
high cost of digital dermoscopic examinations, especially for short-term follow-up patients. Therefore, some policies have been developed to reduce the financial burden on patients, e.g., by providing free of charge three-month followup examinations (19). Finally, Biyik Ozkaya et al. reported that when the histopathological diagnosis is accepted as the gold standard, digital dermoscopy shows $96.6 \%$ sensitivity, $14.9 \%$ specificity, and $47 \%$ diagnostic value, while the evaluation by clinicians shows $100 \%$ sensitivity, $66.66 \%$ specificity, and 95\% diagnostic value. The authors concluded that the clinicians' diagnosis with the ABCD rule is more valuable than diagnoses made by a digital automated dermoscope. Thus, digital dermoscopy is helpful for clinicians to diagnose, but it is not merely enough to diagnose (18). In conclusion, given both the examination and calculation times, the determination of TDS by a handheld analog dermoscope is a very time-consuming process. The present study described a new and time-saving method for determining TDS, by removing the calculation time. It describes a shortcut method to easily see all possible TDSs on a simplified small scale. Thus, this study is the first example in the dermatological literature, in terms of providing a shortcut in the calculation of TDS, without computer aid. The dermoscopic score scale may help both dermatologists and physicians to provide a faster and practical dermoscopic evaluation of nevomelanocytic lesions, especially in clinics without a digital dermoscope.

\section{Footnotes}

Conflict of Interests: The author declares no conflict of interest.

Ethical Approval: Required approval was obtained for this study from the local Ethics Committee of the University of Health Sciences (approval code 10600/11/19).

Funding/Support: The study does not contain any financial or other relationships.

\section{References}

1. Errichetti E, Stinco G. Dermoscopy in general dermatology: A practical overview. Dermatol Ther (Heidelb). 2016;6(4):471-507. doi: 10.1007/s13555-016-0141-6. [PubMed: 27613297]. [PubMed Central: PMC5120630].

2. Ruocco E, Argenziano G, Pellacani G, Seidenari S. Noninvasive imaging of skin tumors. Dermatol Surg. 2004;30(2 Pt 2):301-10. doi: 10.1111/j.1524-4725.2004.30092.x. [PubMed: 14871225].

3. Pehamberger H, Steiner A, Wolff K. In vivo epiluminescence microscopy of pigmented skin lesions. I. Pattern analysis of pigmented skin lesions. J Am Acad Dermatol. 1987;17(4):571-83. doi: 10.1016/s01909622(87)70239-4. [PubMed: 3668002].
4. Ahnlide I, Bjellerup M, Nilsson F, Nielsen K. Validity of ABCD rule of dermoscopy in clinical practice. Acta Derm Venereol. 2016;96(3):36772. doi: 10.2340/00015555-2239. [PubMed: 26351008].

5. Binder M, Puespoeck-Schwarz M, Steiner A, Kittler H, Muellner M, Wolff K, et al. Epiluminescence microscopy of small pigmented skin lesions: Short-term formal training improves the diagnostic performance of dermatologists. J Am Acad Dermatol. 1997;36(2 Pt 1):197-202. doi: 10.1016/s0190-9622(97)70280-9. [PubMed: 9039168].

6. Altamura D, Avramidis M, Menzies SW. Assessment of the optimal interval for and sensitivity of short-term sequential digital dermoscopy monitoring for the diagnosis of melanoma. Arch Dermatol. 2008;144(4):502-6. doi: 10.1001/archderm.144.4.502. [PubMed: 18427044].

7. Kittler H, Pehamberger H, Wolff K, Binder M. Follow-up of melanocytic skin lesions with digital epiluminescence microscopy: Patterns of modifications observed in early melanoma, atypical nevi, and common nevi. J Am Acad Dermatol. 2000;43(3):467-76. doi: 10.1067/mjd.2000.107504. [PubMed:10954658].

8. Gross DA, Zyzanski SJ, Borawski EA, Cebul RD, Stange KC. Patient satisfaction with time spent with their physician. J Fam Pract. 1998;47(2):133-7. [PubMed: 9722801].

9. Zalaudek I, Kittler H, Marghoob AA, Balato A, Blum A, Dalle S, et al. Time required for a complete skin examination with and without dermoscopy: A prospective, randomized multicenter study. Arch Dermatol.2008;144(4):509-13. doi:10.1001/archderm.144.4.509. [PubMed: 18427045].

10. Jaimes N, Dusza SW, Quigley EA, Braun RP, Puig S, Malvehy J, et al. Influence of time on dermoscopic diagnosis and management. Australas J Dermatol.2013;54(2):96-104. doi:10.1111/ajd.12001. [PubMed: 23190378].

11. Kofler L, Egger M, Kofler H. [Sequential digital dermatoscopic imaging. How much time is required per patient?]. Hautarzt. 2014;65(5):450-3. German. doi: 10.1007/s00105-014-2770-y. [PubMed: 24736872].

12. Rogers H, Coldiron BM. Seventy seconds inadequate for a complete skin examination. Arch Dermatol. 2008;144(12):1658-9. author reply 1659-60. doi: 10.1001/archdermatol.2008.501. [PubMed:19075159].

13. Argenziano G, Catricala C, Ardigo M, Buccini P, De Simone P, Eibenschutz L, et al. Dermoscopy of patients with multiple nevi: Improved management recommendations using a comparative diagnostic approach. Arch Dermatol. 2011;147(1):46-9. doi: 10.1001/archdermatol.2010.389. [PubMed: 21242392].

14. Kulatunga-Moruzi C, Brooks LR, Norman GR. Coordination of analytic and similarity-based processing strategies and expertise in dermatological diagnosis. Teach Learn Med. 2001;13(2):110-6. doi: 10.1207/S15328015TLM1302_6. [PubMed: 11302031].

15. Norman GR, Brooks LR. The non-analytical basis of clinical reasoning. Adv Health Sci Educ Theory Pract. 1997;2(2):173-84. doi: 10.1023/A:1009784330364. [PubMed: 12386407].

16. Kittler H, Guitera P, Riedl E, Avramidis M, Teban L, Fiebiger M, et al. Identification of clinically featureless incipient melanoma using sequential dermoscopy imaging. Arch Dermatol. 2006;142(9):1113-9. doi: 10.1001/archderm.142.9.1113. [PubMed: 16982998].

17. Skvara H, Teban L, Fiebiger M, Binder M, Kittler H. Limitations of dermoscopy in the recognition of melanoma. Arch Dermatol. 2005;141(2):155-60. doi: 10.1001/archderm.141.2.155. [PubMed: 15724011].

18. Biyik Ozkaya D, Onsun N, Su O, Arda Ulusal H, Pirmit S. Pitfalls of an automated dermoscopic analysis system in the differential diagnosis of melanocytic lesions. Acta Dermatovenerol Croat. 2014;22(4):278-83.

19. Gadens GA. Lack of compliance: A challenge for digital dermoscopy follow-up. An Bras Dermatol. 2014;89(2):242-4. doi: 10.1590/abd18064841.20142547. [PubMed: 24770499]. [PubMed Central: PMC4008053]. 